\title{
Valparaíso: vulnerabilidad, resiliencia urbana y capital social
}

PAULA KAPSTEIN LÓPEZ

$>$ Escuela de Arquitectura Universidad Católica del Norte, Antofagasta, Chile. pkapstein@ucn.cl MIGUEL ÁNGEL GÁLVEZ HUERTA

> Departamento de Arquitectura Universidad Técnico Federico Santa María, Valparaíso, Chile. miguel.galvez@usm.cl

Universidad de Valparaíso

Facultad de Arquitectura

Revista Márgenes

Espacio Arte Sociedad

Valparaíso: vulnerabilidad, resiliencia urbana y capital social

Diciembre 2014 Vol. 11 № 15

Páginas 25 a 31

ISSN elec. 0719-4463

ISSN imp. 0718-4034

Recepción: Junio 2014

Aceptación: Octubre 2014

\section{RESUMEN}

Partiendo de una reflexión centrada en diagnosticar la alta vulnerabilidad que presentan las ciudades chilenas frente a posibles catástrofes o crisis, se construye una mirada positiva sobre las oportunidades de reconstrucción que se le ofrecen a Valparaíso a partir del incendio del 12 y 13 de abril de 2014, el cual ha puesto a prueba la capacidad de resiliencia de la ciudad y de su gobierno local, de su gente y, en definitiva, de su urbanismo. Se parte de la hipótesis de que estas oportunidades tienen que ver con el mejoramiento de los mecanismos de resiliencia urbana, es decir, el fortalecimiento del capital social y el desarrollo de políticas integrales. Sin embargo, se reconoce que Valparaíso requiere de una metodología específica para dar respuesta a su problemática, por la alta vulnerabilidad medioambiental y urbana que presenta.

PALABRAS CLAVE

vulnerabilidad, capital social, resiliencia urbana, reconstrucción, Valparaíso

\section{Valparaíso: vulnerability, urban resilience and social capital} ABSTRACT

Starting from a reflection considering the high vulnerability of Chilean cities facing natural disasters or crisis, we present a positive view over the opportunities of reconstruction for Valparaiso city after the fire on April $12^{\text {th }}$ and $13^{\text {th }}$ 2014. The fire has put to the test the resilience of the city, its local government, its inhabitants, and ultimately its urbanism. We start from the hypothesis that these opportunities are related to the improvement of urban resilience mechanisms, i.e. the strengthening of social capital and the development of comprehensive policies. However, it's well-known that Valparaíso requires a specific methodology to respond to its problematic due to its high environmental and urban vulnerability.

KEYWORDS

vulnerability, social capital, urban resilience, rebuilding, Valparaíso

\section{Valparaíso: vulnérabilité, résilience urbaine et capital social RÉSUMÉ}

À partir d'une réflexion axée sur le diagnostic de la vulnérabilité des villes chiliennes face à d'éventuelles catastrophes ou crises, nous construisons un regard positif sur les possibilités de reconstruction offertes à Valparaiso depuis l'incendie du 12 et 13 avril 2014, lequel, à tester la capacité de résilience de la ville, celle du gouvernement local, et celle de ses habitants et, en fin de compte, de son urbanisme. Nous partons de l'hypothèse que ces possibilités ont à voir avec l'amélioration des mécanismes de résilience urbaine, c'est-àdire, le renforcement du capital social et le développement de politiques globales. Toutefois, il se reconnaît que Valparaiso a besoin d'une méthodologie spécifique pour répondre à ces problèmes, du a la grande vulnérabilité environnementale et urbaine qu elle présente.

MOTS CLÉS

vulnérabilité, capital social, résilience urbaine, reconstruction, Valparaiso 


\section{INTRODUCCIÓN}

Las ciudades chilenas presentan problemas comunes que dificultan su respuesta ante situaciones de crisis, ya estén éstas referidas a desastres naturales o a fenómenos urbanos dados por crecimientos poblacionales intensos.

El desarrollo del planteamiento general se sustenta en la siguiente hipótesis:

Las ciudades chilenas poseen características referidas a su emplazamiento, a la presencia del paisaje y también a su forma y superficie, que pueden configurar un cuadro de resiliencia física y urbana. Estas mismas características pueden desencadenar el riesgo ante la amenaza si no están acompañadas de una planificación eficaz y preventiva, capaz de anticiparse a ciertos fenómenos críticos. Esta planificación debe considerar al menos tres ejes: políticas y planes que atiendan el desarrollo del capital social y la búsqueda de la equidad, la consideración de criterios geofísicos, medioambientales y climáticos en la definición de usos del suelo, y el diseño de espacios y equipamientos públicos de calidad con buena accesibilidad.

El presente artículo busca construir una reflexión en torno a la reconstrucción de Valparaíso sugiriendo ciertas estrategias desde una base teórica, donde la definición de algunos conceptos como vulnerabilidad urbana permite, primero, realizar un breve diagnóstico de la vulnerabilidad urbana en Chile y del caso puntual de Valparaíso, para luego, fundamentar la necesidad de reforzar el capital social en esta ciudad, presentando un ejemplo del ámbito académico.

\section{ESTADO DE LA CUESTIÓN:}

\section{VULNERABILIDAD DE LAS CIUDADES CHILENAS}

\section{Vulnerabilidad urbana}

La vulnerabilidad urbana es aquella situación crítica dada por problemas en los ámbitos social, físico y urbanístico de la ciudad. Esta situación se caracteriza por su complejidad, la que se da en una superposición de hechos relacionados: la desigualdad social, la degradación del medio físico y la fragmentación del espacio urbano (Kapstein, 2009).

Suárez-Carreño (2005) define la vulnerabilidad como los daños potenciales derivados de una amenaza natural y/o ambiental, en un momento dado, para una población determinada; proponiendo la siguiente relación:

\section{$V=f($ Riesgo, Exposición, Resiliencia)}

En la anterior expresión la vulnerabilidad se representa por la suma de los factores asociados al riesgo, a la exposición de un asentamiento, y a sus elementos de resiliencia. Más adelante, se verá que los elementos de resiliencia deben restarse a la suma de los otros dos.

Asimismo, Ferrando (2003) define los términos riesgo, exposición y resiliencia en relación con los asentamientos urbanos ${ }^{1}$ :

Riesgo: Se define como la probabilidad de una emergencia causada por una amenaza. El nivel de riesgo se establece en relación al tipo de amenaza, al uso del suelo, y a la capacidad intrínseca para soportar o absorber la energía de la amenaza en su proyección. En este sentido el riesgo es el resultado de la interacción entre la diná- mica del medio ambiente natural y el medio ambiente construido.

Exposición: Se refiere a la población amenazada y su relación con la fuente de amenaza. Se puede concebir la exposición como una creación humana en cuanto se concreta, principalmente, en un problema de localización del asentamiento producto del desconocimiento de la dinámica y alcance de los procesos naturales, situación a la que se suman los problemas socioeconómicos y la carencia de cuerpos normativos que regulan el uso del espacio.

Resiliencia: Es la capacidad para soportar y reaccionar ante la emergencia. Depende del nivel riesgo y de las propias fortalezas del asentamiento que le permitan absorber la energía de una amenaza, reorganizándose de otro modo. La resiliencia se puede definir en base a tres parámetros: el tipo de amenaza, el uso de suelo (como un factor externo al propio lugar) y el emplazamiento (como factor inherente al propio asentamiento).

Finalmente, se define una amenaza como cualquier fuente potencial de peligros para una población determinada. Una amenaza puede ser natural o antrópica. En el primer caso, se refiere a aqueIlos procesos naturales que, por la magnitud que pueden alcanzar, son capaces de provocar cambios importantes en el territorio o de alterar su condición de equilibrio de forma más o menos prolongada según sea el tipo de proceso, el área afectada y la recurrencia del fenómeno. Cuando la amenaza es antrópica se refiere a procesos desatados por la intervención del hombre en el territorio, de un modo que va en contra de la naturaleza o directamente en contra de su propio bienestar. Por otra parte, las amenazas, por sí solas, no crean áreas vulnerables; éstas surgen en respuesta a su falta de resiliencia a la amenaza. De este modo que reforzar la resiliencia de áreas urbanas es fundamental para la superación de amenazas de diversa índole.

\section{Breve diagnóstico de la vulnerabilidad urbana en Chile}

Se ha comentado que la vulnerabilidad es la sumatoria de factores asociados a situaciones de riesgo y exposición en los ámbitos social, físico y urbanístico de una ciudad. Este cuadro de vulnerabilidad disminuye si se le restan los elementos de resiliencia. Por poner un ejemplo, en el ámbito social la pobreza puede constituir una situación de riesgo, la falta de iniciativas de participación vecinal puede representar exposición al riesgo, y constituyen factores de resiliencia la existencia de espacios públicos bien articulados y la provisión de equipamientos acordes a las necesidades de los vecinos (Kapstein, 2009). En cuanto a lo medioambiental y/o al soporte geomorfológico de una ciudad, puede constituir un riesgo la posibilidad de un desastre natural sumado a una situación de exposición, la cual se refiere al emplazamiento del barrio en zonas degradadas, contaminadas o cercanas al lugar de la amenaza; y los factores de resiliencia, en este caso, podrían estar referidos al acondicionamiento de los terrenos o a la erradicación del barrio.

De acuerdo a lo anterior, cabe preguntarse por qué las ciudades chilenas son tan vulnerables ante diversas amenazas ya sean éstas naturales o antrópicas.

En primer lugar, el medio urbano posee altas tasas de crecimiento, con ausencia de suelo urbanizado en la periferia, la cual además, presenta una planificación ineficaz para absorber este crecimiento. Por otro lado, la ausencia de inversión en infraestructuras en la periferia de las ciudades impide la oferta de suelo urbano en ellas. La primera consecuencia de ello, es el aumento de precios en las posiciones con mayor centralidad, que se ven expuestas a una alta 
presión inmobiliaria para albergar a clases medias-altas. Las clases medias-bajas y bajas buscan alojamiento en localizaciones alejadas, pero suficientemente conectadas. Las clases más desfavorecidas son expulsadas de este mercado y optan por la informalidad en terrenos altamente vulnerables, generando asentamientos precarios en zonas de riesgo (Kapstein, 2004), con ausencia de equipamientos y muy baja accesibilidad, lo que unido a la pobreza de las personas produce una extrema segregación social. En el Gran Valparaíso esta situación se da en la parte más alta de los cerros.

En cuanto a la legislación urbanística hay grandes vacíos que impiden contar con instrumentos de planeamiento preventivo, los que en el fondo no son otros que instrumentos que conciban la planificación de un modo integral, estratégico y participativo. En especial, se destaca la ausencia de mecanismos de reparcelación para la urbanización de zonas como las afectadas por el incendio en Valparaíso ${ }^{2}$. Mecanismos de este tipo pueden ser de gran ayuda para reconstruir de forma planificada las zonas siniestradas (evitando volver a construir en zonas de riesgo), densificándolas (pues son zonas de baja densidad) y liberando suelo para dotaciones.

Tiene relación con lo anterior, la falta de institucionalidad pública para la reconstrucción de áreas siniestradas, la cual puede considerarse como el conjunto de formas de organización del Estado y las competencias de acción que posibilitan procesos de reconstrucción más eficaces y eficientes (Bresciani, 2012:39). Según Bresciani (2012) la modernización del Estado en esta línea no sólo permitiría responder eficazmente ante los eventos catastróficos, sino que ayudaría también a revertir en forma sustentable los procesos de degradación urbana propios de nuestras ciudades.

Por último, a la falta de institucionalidad frente al riesgo se suma la ausencia de liderazgo de la función pública en el proceso urbanizador, la cual no puede limitarse a un rol subsidiario.

En lo que respecta a la reconstrucción de áreas siniestradas, ésta no puede enfocarse a través de subsidios habitacionales, sin la existencia de políticas y planes creados específicamente para este fin. Como dice Bresciani (2012) hace falta tomar acuerdos para la creación de una Política Nacional de Emergencia y Reconstrucción permanente que incluya una serie de ordenanzas e incluso estrategias generales a seguir de acuerdo al tipo de catástrofe. A dicha Política deberían vincularse los Planes de Reconstrucción que puedan llevarse a cabo en distintas localidades del país en respuesta a situaciones de post catástrofes.

\section{Valparaíso vulnerable}

La ciudad presenta diferentes problemas según se va subiendo desde el Plan a las zonas más altas de sus cerros. Sin pretender ser exhaustivos, a continuación se expone el problema más pregnante en cuanto a la definición de la vulnerabilidad urbana de cada una de estas partes, con el objeto de dejar abierta la reflexión.

En el Plan se da una tugurización de lo edificado. Siendo este problema extremo en ciertas zonas del Almendral, como en las calles cercanas al Terminal de Buses, o en las avenidas Pedro Montt y Francia e incluso en algunas partes de la avenida Argentina; esta degradación del tejido edilicio se da por el abandono total de los propietarios o porque estos deciden rentabilizar sus propiedades de gran tamaño (generalmente incluso con cierto valor patrimonial), subdividiéndolas por dentro para arrendarlas por partes. Este problema es la cara visible de una problemática mayor referida a la gentrificación de las zonas centrales de algunas ciudades en

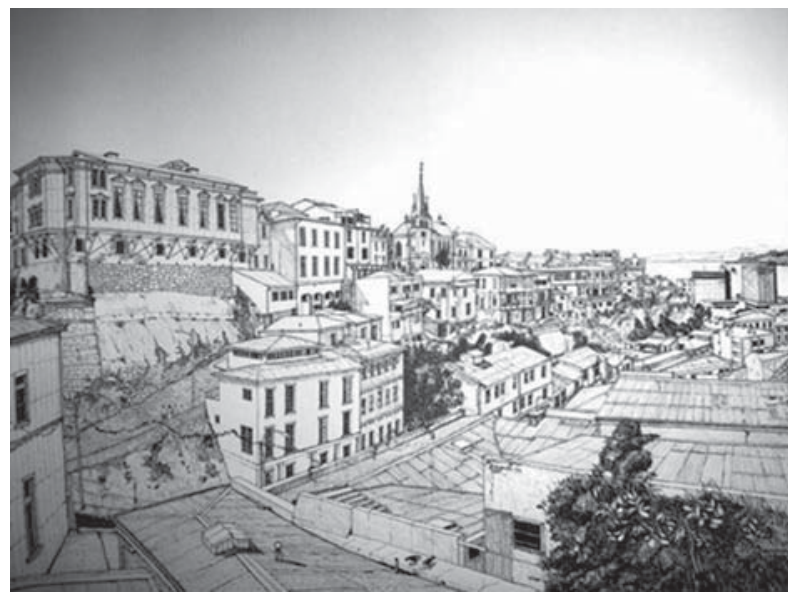

Figura 1. Croquis del cerro Concepción de Vicente Mesina. Fuente: http://festivalartesvalpo.cl/actividades/recorridos-historicos-ilustrados.

nuestro país y también del contexto latinoamericano. Los cambios en los patrones de usos de los centros urbanos y la demanda por vivienda barata en lugares céntricos son algunos de los factores que generan la gentrificación. Sin embargo, también es importante considerar que en Valparaíso los bienes inmuebles de cierta antigüedad sufrieron fuertes daños en el terremoto del año 2010 y esto unido a la falta de programas de rehabilitación del patrimonio, constituye otra de las causas de su obsolescencia.

Se considera que el principal problema de la zona situada entre el Pie de Cerro y el Camino Cintura es la ocupación informal de las quebradas con asentamientos precarios del tipo fondo de quebrada de desarrollo focal (Kapstein, 2004). La ocupación informal de las quebradas no se alude aquí como un problema en sí mismo (en realidad la mayor parte de los cerros de Valparaíso se fue poblando de un modo espontáneo y casi siempre con informalidad), sino más bien por sus consecuencias: la falta de conocimientos técnicos en la autoconstrucción de viviendas sumado a un emplazamiento inadecuado, en zonas de escorrentías o con peligro de deslizamiento de terrenos, definen un cuadro de riesgo ante seísmos que sólo puede subsanarse con programas que atiendan la consolidación de las viviendas que tengan déficits en sus cimientos o en su estructura, considerando un trabajo participativo con los vecinos.

En la zona dada entre el Camino Cintura y la cota doscientos metros se dan dos problemas que unidos forman un cuadro de alto riesgo a amenazas de seísmos como a incendios. Hay, por un lado, vertederos espontáneos o micro vertederos en algunos fondos de las quebradas, los cuales están compuestos principalmente de materias orgánicas, plásticos, ropa, metales, papeles y cartones (Informe del PLADECO, 2002). Además, se presenta el problema de la falta de accesibilidad, que potencia el riesgo de la existencia de los micro vertederos, dificultando su limpieza. La baja accesibilidad dada en las zonas más altas de los cerros se acompaña de grandes carencias en la provisión de equipamientos públicos (de salud, educación y cultura), de modo que las personas que residen aquí deben bajar todos los días al Plan o recorrer importantes distancias en busca de los servicios que requieren.

Finalmente, entre la cota de los doscientos metros y la cota del Camino La Pólvora se da un crecimiento urbano de ocupación dispersa dado mientras el barrio se va constituyendo, con un tipo de 




$>$ Figura 2. Cuadro resumen de diagnóstico sobre aspectos que inciden en la vulnerabilidad de Valparaíso. Fuente: Elaboración propia, 2014.

asentamiento denominado como cima y laderas con un desarroIlo lineal (Kapstein, 2004), que presenta una alta dinamicidad en el tiempo pues el asentamiento crece rápidamente anexionando terrenos nuevos sin haber llegado a densificarse la meseta ni a contar con las infraestructuras básicas, o menos aún, con equipamientos. El riesgo de este tipo de asentamiento está en su falta de consolidación y en la alta insostenibilidad que genera tanto en lo medioambiental como en lo social.

En la figura 2 se presenta un cuadro que resume el diagnóstico realizado.

\section{Valparaíso resiliente}

Tal como en el apartado anterior se ha hecho mención a aspectos relacionados con el riesgo y la exposición a la vulnerabilidad, ahora toca referirse a aquellos elementos referidos a las fortalezas de la ciudad, que son los que construyen la resiliencia de Valparaíso.

Para ello se define el concepto de capital social ya que las fortalezas de Valparaíso hablan de su gente, sus iniciativas y su alta creatividad. Según Valencia et al. (2008) el capital social es importante para el desarrollo económico y social, entendido éste como las relaciones de confianza, redes sociales, organizaciones voluntarias, cívicas u otra serie de factores que lo componen. Además este capital tiene una función dinamizadora de la cohesión social e incluso puede ayudar a reducir la pobreza y la desigualdad, según Valencia, Aguirre y Flórez:

El Estado debe adquirir el compromiso de trabajar en la formulación e implementación de políticas públicas que permitan crear redes entre las organizaciones de base $y$ la sociedad en su conjunto, aumentar la confianza, empoderar a las organizaciones sociales para que construyan y hagan cumplir las normas e integrar a los diversos sectores sociales mediante eslabonamientos que permitan la construcción de consensos alrededor del desarrollo (Valencia, Aguirre y Flórez, 2008:60).

En Valparaíso actualmente es más pregnante el capital humano que el social, el cual puede decirse que aún está en construcción y que surgirá del fortalecimiento de la educación cívica. Falta educar para potenciar redes que ahora son incipientes, para cimentar el cuidado medioambiental y para difundir la solidaridad y el respeto a los otros ${ }^{3}$. En este sentido, hay iniciativas desde el ámbito académico que merece la pena considerar por su incidencia en el fortalecimiento del capital social, como la que se detalla a continuación.

Propuesta de Plaza de los Oficios. Taller de Arquitectura Serrano, Hammersley y Gálvez, impartido durante el segundo semestre de 2014 (Departamento de Arquitectura UTFSM): En este taller avanzado (ciclo profesional de la carrera) se está desarrollando un centro de acogida para emprendedores en los cerros afectados por el incendio de abril de 2014. Para ello se han detectado nueve centralidades con potencialidad para albergar esta agrupación de actividades, las cuales se han propuesto como sitios a los alumnos para el desarrollo de sus proyectos finales (Ver plano de la Figura 3).

Según el catastro de la Seremi de Economía, en Valparaíso hay 1.683 emprendedores en los cerros afectados por el incendio, que lo perdieron todo: local, herramientas, equipos, repuestos, materia- 
les, etc. De estas personas, aproximadamente 1.000 son emprendedores informales (sin registro, pero con actividad comprobada). Además, es sabido que el emprendimiento en los cerros de Valparaíso se realiza en la propia vivienda, en la que se destinan una o varias estancias, así como parte del espacio libre de la parcela, para el desarrollo de la actividad. También es notorio que esta forma de ejercer el emprendimiento tiene múltiples inconvenientes, como la insalubridad y el riesgo de accidentes, pues generalmente las dimensiones de una vivienda rara vez se adaptan a la accesibilidad, necesidades de ventilación e iluminación y demanda de espacio para almacenamiento que requieren los negocios.

Además, por la carencia de equipamientos y de espacio público en la zona siniestrada, los docentes de este taller de Arquitectura entendieron que una agrupación urbana de emprendimientos, además de responder a las necesidades propias de la emergencia, puede constituir un germen de equipamiento comunitario que contribuya a reforzar el tejido social. Esta solución en razón de su escala, según Serrano, Hammersley y Gálvez (2014), posibilitaría al usuario beneficiarse del acceso a servicios como la administración, reutilización de agua, generación de electricidad mediante energías renovables, telecomunicaciones, reciclaje, así como aseos, cocina y lavado comunitarios, que difícilmente pueden conseguirse en negocios individuales.

En cuanto a la definición arquitectónica, durante este curso se considera como una premisa la prefabricación y la construcción modular con el fin de permitir un rápido montaje durante la emergencia, situación para la que se conciben las soluciones. Por ese mismo motivo también se ha planteado lograr la autosuficiencia en la gestión del agua y la energía. Sin embargo, en todos los casos ha prevalecido sobre estas cuestiones la potencialidad del equipamiento propuesto como creador de un espacio para la convivencia.

El quehacer de este taller de arquitectura no sólo resulta de utilidad al momento de fundamentar la importancia que tiene la educación en los procesos de mejoramiento de nuestras ciudades, sino que también ayuda a considerar una proposición teórica aunque concreta, ya que responde a las necesidades de los damnificados por el reciente incendio, permitiendo extraer de ella los planteamientos que puedan servir para elaborar estrategias en la realidad.

\section{REFLEXIONES EN TORNO A LA RECONSTRUCCIÓN DE VALPARAÍSO}

Ya se ha comentado que Bresciani (2012) incide en la urgencia de desarrollar la institucionalidad pública con el fin de no tratar los desastres naturales como eventos extraordinarios. En este sentido, dicho autor propone la creación de una Política Nacional de Reconstrucción. Partiendo de esta idea, se considera que la respuesta a desastres como el incendio de abril pasado en Valparaíso, debe dirigirse a la creación de planes flexibles y de rápida elaboración con una alta participación vecinal ${ }^{4}$, que deberían estar supeditados a una Política Nacional de Reconstrucción.

Por otro lado, es necesario comentar que no es posible reconstruir mediante planes de inversiones sin contar con instrumentos de gestión y de planificación estratégica desarrollados para la emergencia ${ }^{5}$. Ya que es fácil que dichos planes de inversiones se diseccionen y se repartan entre las diversas administraciones del

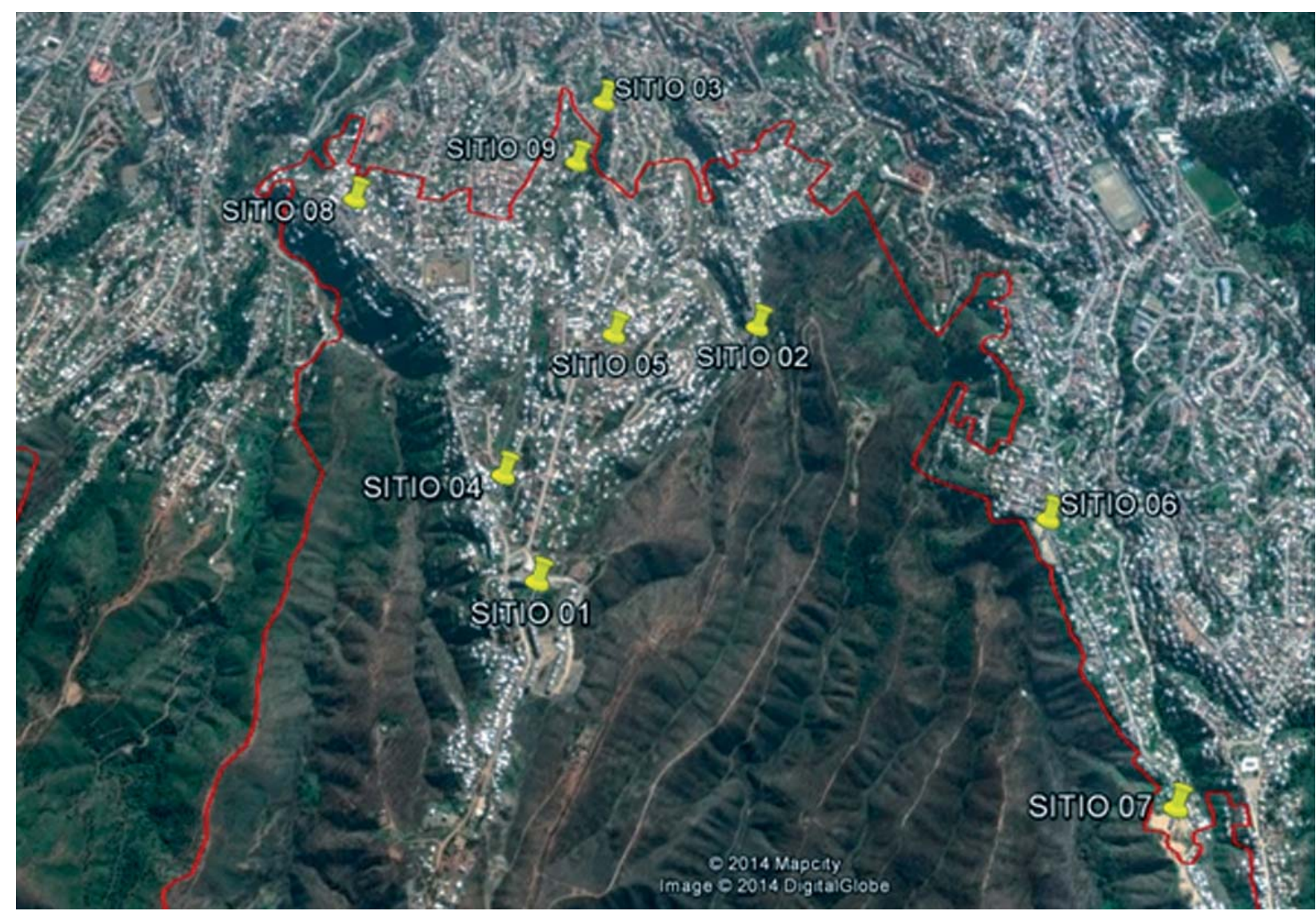

> Figura 3. Plano de emplazamiento de lugares para la Plaza de los Oficios. Fuente: Elaboración de Serrano, P., Hammersley, F. y Gálvez, M. A., sobre imagen de Google Earth, octubre de 2014. Taller avanzado, UTFSM, 2014. 
Estado, las cuales no operan con transversalidad. En Valparaíso hace falta un Plan de Reconstrucción que integre estrategias que puedan ser llevadas a cabo mediante la actuación conjunta e integrada de diversas entidades de la administración pública y por equipos multidisciplinares.

Asimismo, es preciso rescatar el tipo de institucionalidad que la antigua Corporación de Mejoramiento Urbano de Valparaíso (CORMUVAL) ejerció entre 1966 y $1973^{6}$. La Ley orgánica que sustentaba las corporaciones de mejoramiento urbano (CORMU), las facultaba para colaborar y asociarse con las municipalidades y con las empresas privadas en la realización de proyectos de desarrollo y mejoramiento urbano, otorgar créditos para este fin, fiscalizar su realización y fijar dentro de las áreas urbanas los límites de las zonas de mejoramiento urbano y procurar su ordenamiento y desarrollo ${ }^{7}$. Este tipo de gestión urbana está actualmente dando buenos resultados en ciudades como Medellín, con su Empresa de Desarrollo Urbano (EDU), que fue creada en febrero de 2002 como ente jurídico de orden municipal con autonomía administrativa y financiera, con el objeto de ejecutar Proyectos Urbanos Integrales (PUI).

Con el fin de completar las ideas anteriores y pasar de la esfera de la institucionalidad a la del planeamiento, es pertinente citar la definición que aporta Fernández Güell (2007:622) sobre el concepto de planificación estratégica, ya que este tipo de planeamiento, de carácter transversal y de alcance territorial, también podría llevarse a cabo en Valparaíso con buenos resultados:

La planificación estratégica de ciudades es un proceso sistemático, creativo y participativo que sienta las bases de una actuación integrada a largo plazo, que define el modelo futuro de desarrollo, que formula estrategias y cursos de acción para alcanzar dicho modelo, que establece un sistema continuo de toma de decisiones y que involucra a los agentes locales a lo largo de todo el proceso.

La planificación estratégica de ciudades surge en España en la década de los años ochenta porque la planificación urbana tradicional estaba atravesando una crisis, que reflejaba claramente sus limitaciones para satisfacer unas demandas sociales destinadas a conseguir mayor agilidad, participación y transparencia de los procesos de planificación.

De alguna manera, actualmente en Chile está ocurriendo algo similar y las ciudades y sus ciudadanos demandan innovación en los instrumentos de planificación; ahora bien, si además se suma a esto una situación post catástrofe como la de Valparaíso, la demanda se torna en urgencia por encontrar otras respuestas, las cuales dicen relación con lo que se plantea en la hipótesis enunciada en este trabajo.

La planificación porteña debe considerar al menos tres ejes en su desarrollo: capital social, criterios medioambientales y geofísicos en la definición de usos y el diseño de espacios y equipamientos públicos de calidad con buena accesibilidad. Estos lineamientos más o menos básicos podrían dar sustento a cualquier plan de reconstrucción, con distintas jerarquías y acentos en un tema o en otro dependiendo de la realidad local. Por ello, se requiere revisar lo que se ha hecho recientemente en nuestro país, por ejemplo en respuesta al terremoto del 27 de febrero de 2010, y actualizar conceptos y prácticas que puedan ser de utilidad. En este sentido, el Plan de Reconstrucción del Borde Costero de la Región del BíoBío, puede decirse que cuenta con los tres ejes que se mencionan aquí. Este plan tuvo entre sus objetivos redefinir roles, usos del suelo, emplazamientos seguros para la infraestructura y la reurbanización de las dieciocho comunidades costeras siniestradas. Sin embargo, tal como comentó Baeriswyl (2010:62) respecto a los objetivos del Plan de Reconstrucción y al enfrentarse a su coordinación: el mayor desafío de la reconstrucción estará en la recomposición del capital social, destruido luego del 27 de febrero. Por ello, el trabajo participativo con las comunidades afectadas fue el mayor logro de este trabajo.

En el caso anterior, la necesidad de reurbanizar la zona costera arrasada por el tsunami hizo que se diera la posibilidad de la reparcelación. La cual, en el caso de Valparaíso, en su momento pudo y aún puede ser la respuesta en ciertas zonas del área afectada; para ello se requiere de llevar a cabo acciones como la erradicación de las familias de damnificados y su radicación en zonas preparadas para acogerlos durante el tiempo que dure la reurbanización de su zona, la cual debe considerar la elaboración de un Plan Maestro de Reconstrucción que ordene el trazado y defina los usos del suelo, regulando desde la provisión de infraestructuras hasta los equipamientos públicos necesarios. La nueva distribución de terrenos probablemente no permita disponer de viviendas unifamiliares con terreno, pero sí consolidar el barrio, otorgándole lo que antes del incendio no tenía: oportunidades de desarrollo y de cohesión social, accesibilidad, equipamientos de calidad, espacios públicos, la llegada de redes de transporte y viviendas amplias con un diseño innovador en agrupaciones colectivas con espacios comunes. Un Plan de este tipo sólo puede conseguirse con la participación de la ciudadanía puesto que requiere de una visión solidaria: la de construir entre todos barrios y ciudades mejores.

\section{BIBLIOGRAFÍA}

BAERISWYL, S. (2010) "Plan de Reconstrucción Borde Costero Región Bío-Bío”, en Revista CA, N 145, páginas 62-68.

BRESCIANI, L. (2012) “De la emergencia a la política de gestión de desastres: la urgencia de institucionalidad pública para la reconstrucción”. En Brain, I. y Mora, P. (Eds.) Emergencia y reconstrucción: el antes y después del terremoto y tsunami del 27 de febrero en Chile, páginas 39-63, Santiago: Fundación Mapfre y Centro de Políticas Públicas UC.

CLOS, J. (2014) “El poder de las ciudades intermedias" en el VII Foro Urbano Mundial, Medellín, abril del 4 al 11. En http:// wuf7.unhabitat.org/wuf7-reporting-and-conclusions.

FERNÁNDEZ GÜELL, J. M. (2007) “25 años de planificación estratégica". En Ciudad y Territorio Estudios Territoriales, XXXIX (154), páginas 621-637, Madrid.

FERRANDO, F. (2003) “En torno a los desastres naturales: Tipología, conceptos y reflexiones", Revista INVI N 47 , páginas 13-27, Santiago: Facultad de Arquitectura y Urbanismo de la Universidad de Chile.

INFORME PLADECO (2002) Municipalidad de Valparaíso. Consultado en http://www.munivalpo.cl/transparencia/archivos/ plan_desarrollo_comunal/PLADECO.pdf.

KAPSTEIN, P. (2009). La Periferia Interior. Un problema de vulnerabilidad no resuelto por el planeamiento urbano en Chile, Tesis doctoral leída en la Escuela Técnica Superior de Arquitectura de la Universidad Politécnica de Madrid. Tutor: Agustín Hernández Aja. 
"Análisis de asentamientos precarios en Valparaíso y su incidencia en el crecimiento urbano", Revista INVI N 49 , páginas 83-101, Santiago: Facultad de Arquitectura y Urbanismo de la Universidad de Chile.

RAPOSO, A., VALENCIA, M. (2004) “Modernidad, Diseño Urbano y Utopía: Notas sobre el fundamento político de las acciones de Remodelación Urbana en Santiago. El caso de la CORMU 1966- 1973", Revista de Urbanismo, № 9, páginas 116-140, Universidad de Chile.

SERRANO, P., HAMMERSLEY, F., GÁLVEZ, M .A. (2014) Documento de elaboración interna para desarrollo del Taller avanzado $2^{\text {do }}$ semestre, Departamento de Arquitectura UTFSM, Valparaíso.

SUÁREZ-CARREÑO, L. (2005) "Vulnerabilidad territorial: un reto para cooperación en hábitat". En Jornadas de Habitabilidad Básica y Desarrollo, AECl, Madrid.

VALENCIA, G., AGUIRRE, M., y FLÓREZ, J. (2008) "Capital social, desarrollo y políticas públicas en Medellín, 2004-2007”. En Estudios Políticos, N 32, Instituto de Estudios Políticos, Universidad de Antioquia, páginas 53-83.

\section{NOTAS}

1 Definiciones elaboradas por los autores a partir de los conceptos presentados en el artículo de Francisco Ferrando A. "En torno a los desastres naturales: Tipología, conceptos y reflexiones" en la Revista INVI No 47, editada por la Facultad de Arquitectura y Urbanismo de la Universidad de Chile, mayo de 2003.

2 En España, por ejemplo, el desarrollo y ejecución de una actuación urbanística se lleva a cabo mediante una Junta de Compensación, que reparcela, urbaniza y distribuye entre los propietarios las cargas (infraestructura, espacios públicos, equipamientos y reserva de suelo para vivienda social) y los beneficios (aprovechamiento urbanístico), de acuerdo a las indicaciones de un Plan Parcial. Referencia: A nivel estatal, se refleja en el Reglamento de Planeamiento (1978). Tras la descentralización de las competencias en materia de urbanismo, cada comunidad autónoma tiene su propia legislación (en Madrid Ley 9/2001).

3 El Urbanismo Cívico Pedagógico implantado en Medellín por su Municipalidad se basó en los siguientes pilares: COMUNICAR, CONECTAR, EDUCAR y MOTIVAR. Esta fórmula se está haciendo realidad desde hace varios años, permitiendo al Estado reforzar redes sociales y llevar a cabo proyectos en conjunto con la ciudadanía.

4 Un buen ejemplo de este tipo de instrumento de planificación estratégica lo constituye el Plan de Reconstrucción del Borde Costero Región Bío-Bío (2010), que fue coordinado por Sergio Baeriswyl.

5 Actualmente en Valparaíso se está ejecutando un Plan de Inversiones para la Reconstrucción, aunque hay propuestas válidas y bien elaboradas como la del Plan Maestro para la Reconstrucción y Planificación de Valparaíso (2014), realizada por la Comisión Especial de Reconstrucción del Colegio de Arquitectos de Valparaíso.

6 Raposo y Valencia (2004) desarrollan una tesis sobre el proyecto urbano en Chile, como respuesta a una cultura urbanística y unas prácticas estatales que propiciaban el desarrollo de esta cultura en los gobiernos de Frei y de Allende.
7 Artículo $2^{\circ}$ del Título I de la Ley Orgánica de la Corporación de Mejoramiento Urbano (CORMU).

\section{AGRADECIMIENTOS}

Desde fines de abril del presente año, uno de los autores ha venido participando junto con colegas de las universidades de la región en las reuniones del Comité Asesor Técnico para la Comisión Especial del Senado "Catástrofe e Incendio de Valparaíso", presidida por el senador Antonio Horvath. En una iniciativa sin precedentes, durante cuatro meses las distintas escuelas de Arquitectura de la V Región han debatido una postura común frente a la catástrofe, que permitiera hacer llegar una serie de recomendaciones al poder legislativo. Acompañadas de diversas presentaciones a la comunidad académica (en la Escuela de Derecho de la UV y en la UTFSM), los trabajos de la comisión concluyeron el pasado octubre con una exposición de las escuelas ante el Senado, quedando únicamente pendiente la publicación de un informe de conclusiones. Alguna de las reflexiones que se desgranan en este artículo es deudora de las aportaciones de nuestros compañeros de Academia en dichas reuniones. Por ello, quisiéramos mostrar nuestro agradecimiento a todos ellos. 